\title{
EUS Accuracy Against MRCP for Detection of
}

\section{Pancreaticobiliary Lesions}

\author{
Mustafa Zanyar Akkuzu*, Engin Altıntaş, Serkan Yaraş, Osman Özdoğan, Enver Ucbilek, Fehmi \\ Ates, Ferzan Aydın, Hatice Rızaoğlu Balcı, Yaren Dirik, Orhan Sezgin
}

Mersin University, Faculty of Medicine, Department of Gastroenterology

\begin{abstract}
Anatomic alterations due to the hepatobiliary system pathologies such as stenosis, dilatation, stone, and tumor can be suspected by clinical symptoms, laboratory tests and eventually confirmed by imaging methods. Both Magnetic Resonance Colangiopathy (MRCP) and Endoscopic Ultrasonography (EUS) are diagnostic tools for etiologic assessment of extrahepatic cholestasis. We aimed to compare MRCP and EUS, about the superiority in diagnostic terms, in our patient group with hepatobiliary system disorders.

The results of 135 patients who underwent both MRCP and EUS in the Hospital Gastroenterology Clinic of Mersin University Medical Faculty between 2010-2018 were included in the study. After reviewing the MRCP and EUS reports; stone, tumor and pancreatitis were evaluated and analyzed.

Of the patients, $71(52.6 \%)$ were male and $64(47.4 \%)$ were female. The mean age of males was $60.5 \pm 15.49$ years and that of females was $61.2 \pm 14.25$. The age spectrum was $23-91$ years. In $97(71.9 \%)$ of patients, MRCP and EUS were reported in the same way and both imaging methods led to the correct diagnosis. There were $38(28.14 \%)$ patients with different diagnoses and the total cases with stone, tumor and pancreatitis were evaluated and compared. The sensitivity of EUS for the stone was $88.9 \%$ and that of MRCP was $81.5 \%$. The sensitivity of EUS was $92.5 \%$ and that of MRI was $66 \%$, in the diagnosis of the tumor and IPMN. In pancreatitis, the sensitivity of EUS was $89.7 \%$ and MRCP was $72.4 \%$.

EUS is a better diagnostic tool for the diagnosis of choledocholithiasis, tumor and pancreatitis than MRCP.
\end{abstract}

Key Words: Endoscopic Ultrasonography, Magnetic Resonance Imaging, Pancreatobiliary Imaging

\section{Introduction}

Approximately 1-1.5 liters of bile are synthesized daily, from the liver and secreted through the biliary tract to the duodenum. The occlusion in the biliary tract for any reason (such as stone, tumor, stenosis, inflammation, compression) is defined as mechanical icterus. In this case, clinical symptoms such as yellowing of sclera, darkening in urine color, paling in stool color, abdominal pain and fever can occur in patients. Laboratory findings and imaging methods are used in diagnosis (1).

Pathologies such as stenosis, enlargement, stone and tumor in biliary tract are detected with imaging methods. Ultrasonography (USG), Endoscopic ultrasonography (EUS), Computed Tomography (CT), Magnetic Resonance Cholangiopancreatography (MRCP) and Endoscopic retrograde cholangiopancreatography (ERCP) can be used to detect biliary tract pathologies. However, in the era of which, the
ERCP is used only for therapeutic purposes and not for diagnosis, alternatively EUS can be performed (2). In the literature, the sensitivity and specificity of USG was found to be above $90 \%$ in detecting biliary tract pathologies. The sensitivity and specificity of CT in detecting biliary tract stones are lower than in USG. CT stands out in the diagnosis, staging and assessment of vascular involvement of the pancreatic tumors'; the view of compression effect of tumoral mass can be shown. MRCP is the most important noninvasive imaging method in detecting biliary tract diseases and its sensitivity and specificity rates are $95 \%$ and $89 \%$, respectively (3). We planned a retrospective study to compare the MRCP and EUS, to determine their superiority in terms of diagnosis, in our patient group with hepatobiliary disorders.

\section{Material and Methods}

Totally, 135 cases were evaluated, to whom MRCP and EUS were performed together at Mersin 
University Faculty of Medicine Gastroenterology Clinic between 2010-2018, the reports were gathered from the hospital data records and their results were compared. These cases were related to the liver, biliary tract and pancreas. In order to understand which imaging method made the correct diagnosis, ERCP, surgery report and the evaluation made with the pathology result were accepted as the gold standard and MRCP and EUS results were compared with them. After examining the reports; sensitivity data for stones, tumors and pancreatitis were evaluated and analyzed. Our study was retrospective and patient consents were obtained during EUS and ethical committee approval was obtained by the clinical research ethics committee of our university, numbered 117 decision 2019. SPSS for Mac Version 21.00 (SPSS Inc., Chicago, IL., USA) package program was used to calculate the average and standard deviation values and rates in the statistical analysis of the data obtained at the end of the study. Chisquare test was used for analysis of categorical variables, and student $t$ test was used for analysis of continuous variables.

\section{Results}

Of the patients we evaluated $71(52.6 \%)$ were male and $64(47.4 \%)$ were female. The average age of the men was $60.5 \pm 15.49$ and the average age of the women was $61.2 \pm 14.25$ years. The range of age in our patients was 23-91 years. In 97 of the cases $(71.9 \%)$ MRCP and EUS, both were reported in the same way and both imaging methods gave the correct results. There were 38 $(28.14 \%)$ patients with different diagnoses. The cases with stones, tumors and pancreatitis were evaluated in detail and compared.

Among the patients with the diagnosis of biliary stone, ten cases with stone could be seen in both imaging methods. With EUS, only three of the total patients were undetectable, twenty-four cases with stone were detected. Biliary stones could be detected in 22 patients by MRCP but not in 5 patients. Stones could be detected by neither MRCP nor EUS, in two patients. Cases not seen in EUS were seen by MRCP and cases not seen in MRCP were seen by EUS.

Forty-nine of 53 cases with tumors or IPMN were able to be visualized with EUS, but four were not. In 17 of 18 patients diagnosed with malignancy, no lesions were detected in MRCP, but in EUS. However, one patient did not have any lesions on both examinations. MRCP made a clear diagnosis in thirty-one of the patients, only four were skeptical and eighteen cases were not seen by MRCP. In 30 cases, both imaging methods gave the correct result. One case was not seen in both imaging methods and was eventually diagnosed by ERCP.

Twenty-six of 29 patients with pancreatitis could be seen by EUS, but three were not. Twenty-one of them were seen by MRCP and eight of them could not be seen. In 17 pancreatitis, both EUS and MRCP had the correct diagnosis.

In $5(3.7 \%)$ patients, both EUS and MRCP were insufficient in diagnosis. Of them 2 patients $(1.5 \%)$ could not show the stone, the remaining 3 $(2.2 \%)$ were combined cases and only one of the diagnoses could be detected. In 14 cases, both imaging methods were completely normal and gave the correct result. The remaining cases were diagnoses such as cyst, Oddie fibrosis, pancreatic divisium and both imaging methods gave the correct and the same result.

Among the total 135 patients, the lesion or stone were not visualized with EUS, in 3 cases with stones, 3 cases with pancreatitis, 4 cases with tumors or IPMNs. With MRCP, 18 cases with tumors or IPMNs could not be displayed and 4 cases were reported as suspected, but not making the diagnosis clearly. 5 cases with stones, 8 cases with pancreatitis and one pancreatic divisium were not fully visualized by MRCP.

The sensitivity rates for EUS and MRCP for stone diagnosis were $88.88 \%$ and $81.5 \%$, respectively. The sensitivities of EUS and MRCP, in the diagnosis of tumor and IPMN were $92.5 \%$ was $66 \%$, respectively. In the diagnosis of pancreatitis, the sensitivities of EUS and MRCP were $89.7 \%$ and $72.4 \%$, respectively. EUS was more significant than MRCP in detecting stones, tumors and pancreatitis (Table 1).

Considering the total of 135 patients, EUS failed in diagnosis in $10(7.4 \%)$ cases and eventually made the correct diagnosis in 125 (92.6\%) of cases. MRCP was able to give the correct diagnosis in $104(77 \%)$ patients, but didn't yield the correct diagnosis in $31(23 \%)$ patients. EUS was found to be a superior diagnostic tool than $\operatorname{MRCP}(\mathrm{p}<0,016)$.

\section{Discussion}

We compared the diagnostic capabilities of EUS and MRCP in detecting choledocholithiasis in suspected patients. The most emerged advantage of MRCP are its completely non-invasive nature, and possibly being highly tolerated compared to

East J Med Volume:25, Number:4, October-December/2020 
Table 1. Sensitivity Comparison

\begin{tabular}{lccc}
\hline & EUS Sensitivity & MRCP Sensitivity & $\mathrm{P}<0,05$ \\
\hline Stone & $\% 88,9$ & $\% 81,5$ & 0,016 \\
Tumor + IPMN & $\% 92,5$ & $\% 66$ & 0,001 \\
Pancreatitis & $\% 89,7$ & $\% 72,4$ & 0,016 \\
\hline
\end{tabular}

EUS, especially by high-risk patients such as the elderly or severe patients. EUS provides very high resolution images due to the proximity of the ultrasound transducer to the internal structures. This higher resolution (higher than MRCP), makes the EUS even more sensitive in the diagnosis of small bile stones. In our study, the sensitivity of EUS (88.9\%) for detecting choledocholithiasis was higher than MRCP (81.5\%).

The exact prevalence of gallstones in the general population is difficult to determine, because the gallstones often have asymptomatic nature. Only one-third of the gallstones cause symptoms or complications, such as choledocholithiasis (4-6). ERCP must be reserved only for therapeutic purposes, due to its own risk of complication. EUS and MRCP have become the preferred method for the diagnosis of choledocholithiasis (4-6). Despite advances in MRCP techniques for visualizing biliary abnormalities, its role is limited, and contrast agents can be needed and a histological diagnosis is not provided. Thus, EUS has emerged as an important tool in the assessment of biliary disease.

In studies comparing MRCP and EUS, in the diagnosis of choledocholithiasis, (with the ERCP as the gold standard) EUS sensitivity was found to be higher than MRCP. There is now a lack of consensus on the optimal non-invasive strategy for patients with suspected choledocholithiasis. (713). In our study, in accordance with the literature, the sensitivity of EUS for stone was $88.9 \%$ and MRCP was $81.5 \%$. However, since EUS and MRCP are not made exactly at the same time, there is a possibility that the bile stone can leave the biliary system, through the papilla into the duodenum, spontaneously.

Many studies comparing EUS and MRCP in idiopathic acute pancreatitis have shown that EUS has higher diagnostic yields in idiopathic pancreatitis than MRCP. For idiopathic acute pancreatitis, EUS should be considered as the first choice. Biliary diseases such as cholelithiasis, choledocholithiasis, microlithiasis and biliary sludge are considered the leading cause of idiopathic acute pancreatitis. Microlithiasis, defined as the presence of stones $<5 \mathrm{~mm}$ in diameter, is thought to cause unexplained pancreatitis attacks in $75 \%$ of patients without any history of cholecystectomy. It demonstrated that EUS has a better diagnostic efficiency in detecting bile duct stones less than $5 \mathrm{~mm}$ compared to MRCP (14-20). In our study, the sensitivity of EUS in acute pancreatitis was $89.65 \%$ and MRCP was $72.4 \%$. However, since EUS and MRCP are not performed at the same time, it should be considered that the possibility of some patients with acute pancreatitis, pancreatic inflammation may be diminished, already. The major weakness of our study was due to retrospective design.

Pancreatic divisium is a congenital anomaly resulting from the dorsal and ventral pancreatic glands opened into the duodenum separately, and its prevalence is $5 \%$ to $14 \%$ of the population $(21,22)$. In our study, MRCP was not able to diagnose pancreatic divisium in one patient, whereas EUS provided imaging in all pancreatic divisium cases.

Intraductal papillary mucinous neoplasms (IPMNs) of the pancreas have been well recognized since 1982, date of the first report by Ohashi et al (23). They consist of pancreatic tumors characterized by papillary proliferation of the ductal epithelium. A study from Father and friends (24); compared to EUS and magnetic resistance cholangiopancreatography (MRCP), concluded that EUS is the most effective in distinguishing benign IPMNs from malignant tumors. Fernández-Esparrach et al. showed that EUS -FNA has a sensitivity of $82 \%$ in the diagnosis of IPMNs (25). Compared with transabdominal US and MRI in malignant cancers of the pancreas, EUS (98\%) has a superior parenchymal resolution. In the study, abdominal ultrasonography has the sensitivity of $75 \%$, that of CT was $80 \%$ and MR angiography $89 \%$. EUS was even much better, especially in tumors smaller than $3 \mathrm{~cm}$ (26). EUS is also reliable for localization of pancreatic neuroendocrine tumors (sensitivity, $82 \%$ and specificity, 95\%) and is quite accurate in estimating the true size of these tumors (2 $\mathrm{mm}$ deviation between EUS and surgical pathology) (27). Endoscopic ultrasonography (EUS) is also an effective method in the preoperative staging of ampullary 
neoplasms. It provides detailed information about the size of the tumor, the depth of invasion; and mucosal, parenchymal, vascular, ductal and nodal changes in the lesion area. EUS helps to decide on local or radical treatment. EUS is more sensitive than CT and MRI in local staging of ampullary tumors (EUS 78\%, BT 24\%, MR 46\%) (28). Despite the ongoing development of other crosssectional imaging methods such as dynamic MR, EUS still plays a leading role in the search for pancreaticobiliary diseases. EUS is the most accurate method for detecting small $(<3 \mathrm{~cm})$ pancreatic lesions that including NETs and ampullary neoplasms and the best method to identify vascular infestation in pancreas and periampullary tumors. The ability in yielding pancreatic tissue with EUS-FNA is very important in clinical decision making in patients with pancreatic cancer; it exhibits excellent sensitivity and specificity and looks safe when performed by experienced endosonographers (29). In our study, EUS was better than MRCP in detection of pancreatic tumors, ampullary tumors and IPMNs; sensitivity of EUS was $92.5 \%$ and that of MRCP was $66 \%$, in parallel with the literature. Although $\mathrm{MR}$ is useful in detecting and identifying pancreatic masses, we conclude that it should not be the first choice in the diagnosis and staging of pancreatic cancers.

As a result, EUS is a better diagnostic tool than MRCP for the diagnosis of choledocholithiasis, and that of tumor and pancreatitis. EUS has also advantage of histologic yielding in the diagnosis of all pancreatobiliary diseases, especially in tumor cases. Moreover, this superiority gets more eminance with the experience of EUS practitioner. We think that EUS practitioners are more successful in making a diagnosis because the lesions can be shown dynamically, because of the real time nature of EUS.

\section{References}

1. Bhat M, Romagnuolo J, da Silveira E et al. Randomised clinical trial: MRCP-first vs. ERCP-first approach in patients with suspected biliary obstruction due to bile duct stones Aliment Pharmacol Ther 2013; 38: 1045-1053.

2. Moon JH, Cho YD, Cha SW et al. The detection of bile duct stones in suspected biliary pancreatitis: comparison of MRCP, ERCP, and intraductal US Am J Gastroenterol 2005; 100: 1051-7. 4.

3. Hekimoglu K, Ustundag Y, Dusak A et al. MRCP vs. ERCP in the evaluation of biliary pathologies: review of current literature J Dig Dis 2008; 9: 162-169.

4. Smith ZL, Meiselman MS. Calculous biliary disease, Conn's current therapy Philadelphia: Elsevier Saunders 2013; 500-503.

5. Freitas ML, Bell RL, Duffy AJ. Choledocholithiasis: evolving standards for diagnosis and management World J Gastroenterol 2006; 12: 3162-3167.

6. Vázquez-Sequeiros E, Tamargo F, BoixedaMiquel D, Milicua JM. Diagnostic accuracy and therapeutic impact of endoscopic ultrasonography in patients with intermediate suspicion of choledocholithiasis and absence of findings in magnetic resonance cholangiography Rev Esp Enferm Dig 2011; 103: 464-471.

7. Makmun D, Fauzi A and Shatri H. Sensitivity and Specificity of Magnetic Resonance Cholangiopancreatography versus Endoscopic Ultrasonography against Endoscopic Retrograde Cholangiopancreatography in Diagnosing Choledocholithiasis: The Indonesian Experience Clin Endosc 2017; 50: 486-490.

8. Alhayaf N, Lalor E, Bain V, McKaigney J, Sandha GS. The clinical impact and cost implication of endoscopic ultrasound on use of endoscopic retrograde cholangiopancreatography in a Canadian university hospital. Can J Gastroenterol 2008; 22: 138-142.

9. Aubé C, Delorme B, Yzet T, et al. MR cholangiopancreatography versus endoscopic sonography in suspected common bile duct lithiasis: a prospective, comparative study AJR Am J Roentgenol 2005; 184: 55-62.

10. Giljaca V, Gurusamy KS, Takwoingi Y, et al. Endoscopic ultrasound versus magnetic resonance cholangiopancreatography for common bile duct stones. Cochrane Database Syst Rev. 2015:CD011549.

11. Mesihović R, Mehmedović A. Better noninvasive endoscopic procedure: endoscopic ultrasound or magnetic resonance cholangiopancreatography? Med Glas (Zenica) 2019; 16: 40-44.

12. De Castro VL, Moura EG, Chaves DM et al. Endoscopic ultrasound versus magnetic resonance cholangiopancreatography in suspected choledocholithiasis: A systematic review. Endosc Ultrasound 2016; 5: 118-128.

13. Garrow D, Miller S, Sinha D, Conway J et al. Endoscopic ultrasound: a meta-analysis of test performance in suspected biliary obstruction. Clin Gastroenterol Hepatol. 2007; 5: 616-623.

14. Ortega AR, Gómez-Rodríguez R, Romero M, Fernández-Zapardiel S, Céspedes Mdel M, Carrobles JM . Prospective comparison of

East J Med Volume:25, Number:4, October-December/2020 
endoscopic ultrasonography and magnetic resonance cholangiopancreatography in the etiological diagnosis of idiopathic acute pancreatitis. Pancreas 2011; 40: 289-294.

15. Wan J, Ouyang Y, Yu C, Yang X, Xia L, Lu, $\mathrm{N}$. Comparison of EUS with MRCP in idiopathic acute pancreatitis: a systematic review and meta-analysis. Gastrointest Endosc 2018; 87: 1180-1188.

16. Vila JJ. Endoscopic ultrasonography and idiopathic acute pancreatitis. World J Gastrointest Endosc 2010; 2: 107-111.

17. Wilcox, C.M., Seay, T., Kim, H. et al. Prospective endoscopic ultrasound-based approach to the evaluation of idiopathic pancreatitis: causes, response to therapy, and long-term outcome. Am J Gastroenterol 2016; 111: 1339-1348.

18. IAP/APA evidence-based guidelines for the management of acute pancreatitis. Pancreatology 2013; 13: 1-15.

19. Wilcox, C.M., Varadarajulu, S., and Eloubeidi M. Role of endoscopic evaluation in idiopathic pancreatitis: a systematic review. Gastrointest Endosc 2006; 63: 1037-1045.

20. Kondo S, Isayama, $H$, Akahane $M$ et al. Detection of common bile duct stones: comparison between endoscopic ultrasonography, magnetic resonance cholangiography, and helical-computedtomographic cholangiography. Eur J Radiol 2005; 54: 271-275.

21. Quest, L. and Lombard, M. Pancreas divisum: opinio divisa. Gut 2000; 47: 317-319.

22. Manfredi R, Costamagna G, Brizi MG et al. Pancreas divisum and "santorinicele": diagnosis with dynamic MR cholangiopancreatography with secretin stimulation. Radiology 2000; 217: 403-408.

23. Ohashi K, Murakami Y, Takekoshi T. Four cases of mucin producing cancer of the pancreas on specific findings of the papilla of Vater. Prof Dig Endosc 1982; 20: 348-351.

24. Baba T, Yamaguchi T, Ishihara $T$ et al. benign from malignant intraductal papillary mucinous tumors of the pancreas by imaging techniques. Pancreas 2004; 29: 212-217.

25. Fernández-Esparrach G, Pellisé M, Solé M et al. EUS FNA in intraductal papillary mucinous tumors of the pancreas. Hepatogastroenterology 2007; 54: 260-264.

26. Palazzo L, Roseau G, Gayet B, Vilgrain V, Belghiti J, Fékéte F, Paolaggi JA. Endoscopic ultrasonography in the diagnosis and staging of pancreatic adenocarcinoma. Results of a prospective study with comparison to ultrasonography and CT scan. Endoscopy 1993; 25: 143-150.

27. Michl P, Pauls S, Gress TM. Evidence-based diagnosis and staging of pancreatic cancer. Best Pract Res Clin Gastroenterol 2006; 20: 227-51.

28. Cannon ME, Carpenter SL, Elta GH et al. EUS compared with CT, magnetic resonance imaging, and angiography and the influence of biliary stenting on staging accuracy of ampullary neoplasms. Gastrointest Endosc 1999; 50: 27-33.

29. Fusaroli P, Kypraios D, Caletti G, and Eloubeidi MA. Pancreatico-biliary endoscopic ultrasound: A systematic review of the levels of evidence, performance and outcomes. World J Gastroenterol 2012; 18: 4243-4256. 